\title{
Quality of service implications of power control and multiuser detection-based cross-layer design
}

\author{
Ulrike Korger ${ }^{1 *}$, Christian Hartmann ${ }^{1}$, Katsutoshi Kusume ${ }^{2}$ and Joerg Widmer ${ }^{3}$
}

\begin{abstract}
In order to allow for dense spatial reuse in wireless ad hoc networks, multiple access interference must be dealt with. This calls for advanced physical layer techniques, such as multiuser detection (MUD) or power control. However, these techniques can only be efficiently applied to ad hoc networks when they are part of a joint physical layer (PHY) and Medium Access Control (MAC) cross-layer design (CLD). In order to better understand both, the potential but also the limits of handling interference by means of MUD and power control, respectively, in this article we provide a comprehensive comparison between MUD-based and power control-based CLDs. We study the behavior of both approaches in terms of throughput, delay, as well as fairness in scenarios with high and low user densities, respectively. To provide more detailed insight in the interaction between MAC and PHY, we separate for each approach the throughput results into gains achieved solely by the MAC layer and by the PHY layer, respectively. These results highlight, among other aspects, some fundamental disadvantage of power control in distributed environments. We conclude that multiuser-based approaches are significantly more beneficial in ad hoc scenarios than power control-based schemes.
\end{abstract}

\section{Introduction}

Dense ad hoc networks typically suffer from multiple access interference (MAI). A well known approach to battle this interference is to block users in the vicinity of a communication pair, e.g., by applying an RTS/CTS signaling as in the IEEE 802.11 protocol, which, however, obviously limits the spatial reuse significantly. When targeting a denser spatial reuse, more sophisticated means for dealing with interference are required. Some of the approaches suggested in the literature are multiuser detection (MUD) and power control. While the application of those approaches is basically well understood in cellular environments, it still constitutes a challenge to efficiently apply them in ad hoc networks, where no infrastructure is available. Therefore distributed protocols are required, which interact closely with the physical layer to enable MUD or power control, respectively. Hence it is not sufficient to consider the physical layer only. We rather have to look at joint PHY/MAC cross-layer designs (CLDs) in which the

\footnotetext{
* Correspondence: ulrike.korger@tum.de

${ }^{1}$ Institute of Communication Networks, Technische Universität München, Arcisstr. 21, 80290 Munich, Germany

Full list of author information is available at the end of the article
}

MAC protocol is specifically designed to support the respective physical layer technique.

Power control, which has been successfully applied to cellular networks, has received considerable attention in the field of ad hoc networks as well. It has been combined with specific MAC protocols to apply it in distributed ad hoc networks for MAI suppression by many authors, e.g., [1], [2], [3].

A different physical layer technique, which also has received considerable attention in the literature is MUD, applied at the receiver side [4]. An MUD receiver detects interfering streams to subtract their interference contribution from the received signal, thus canceling MAI. The complexity of MUD generally increases exponentially with the number of detected streams, i.e., with the number of receiver branches [5]. However, algorithms with reduced complexity are available, which achieve similar performance [6]. MUD has also been investigated by several authors in the context of ad hoc networks by combining it with appropriate MAC protocols, which enable MUD operation on the physical layer, e.g., [7], [8], [9].

We are interested in the capability of both, power control-based and MUD-based cross-layer solutions. Both approaches aim at increasing the spatial reuse by

\section{SpringerOpen ${ }^{\circ}$}

(C) 2011 Korger et al; licensee Springer. This is an Open Access article distributed under the terms of the Creative Commons Attribution License (http://creativecommons.org/licenses/by/2.0), which permits unrestricted use, distribution, and reproduction in any medium, provided the original work is properly cited. 
means of MAI suppression. However, the two physicallayer techniques differ fundamentally in the way they each treat MAI as well as in the required interaction with the MAC protocol. While the performance of both techniques is well understood on the physical layer alone, a detailed numerical comparison between power control-based and MUD-based CLDs is not yet available.

In this article we start out with a detailed review and discussion of available CLDs for both power control and MUD. Eventually, we are concerned with the Quality of Service (QoS) achieved with the different CLDs. For this purpose we thoroughly investigate two representative CLDs, one for each physical layer approach. Namely, we compare the Progressive BackOff Algorithm (PBOA) approach [3], a good representative for power controlbased CLD, to the MUD-MAC CLD that was presented in [9]. Both protocols are based on a similar time slotted frame structure and are each designed to support the respective physical-layer technology. We assess and compare the QoS of both schemes by means of extensive system simulations in terms of data throughput as well as delay. However, we also consider the fairness of both schemes as an additional important QoS aspect.

Parts of the results presented here have earlier been published in [10] and [11].

The remainder of this article is organized as follows. We start with a discussion of power control in ad hoc networks and power control-based CLDs in Sect. II, before we summarize the PBOA that serves as a comparison scheme for our MUD-MAC protocol in Sect. III. Then we introduce the functional principle of MUD and discuss MUD-based CLDs from the literature in Sect. IV. The MUD-MAC CLD, as our representative MUD CLD, is described in Sect. V. We explain the applied delay and fairness measures in Sect. VII. Throughput, fairness, and delay results are presented in Sect. VIII for random networks. Section IX draws the conclusions.

\section{Power control-based medium access}

In wireless ad hoc networks, multiple nodes simultaneously try to access the channel without any central control instance. This poses major challenges for power control, since all transmitters must decide on the power level they want to apply in an upcoming transmission in a fully distributed way.

\section{A. Power control functional principle}

In order to agree on individual transmission powers, nodes start gaining information about the interference situation in their vicinity. Assuming this information is somehow obtained, they adapt their individual power levels such that they, on the one hand, are able to reach their associated partners and, on the other hand, avoid overwhelming other receivers with interference. If this is not possible, e.g., due to certain distance relationships, some transmitters have to abstain from transmitting.

Summarizing, this poses three challenges on power control-based CLD in ad hoc networks, namely

(1) Achieve the information about the interference situation in a fully distributed way.

(2) Appropriately adapt power levels.

(3) Realize blocking situations beforehand.

\section{B. Overview of power control-based CLDs}

In the following, we present a State-of-the-Art overview for power control-based CLDs in wireless ad hoc networks. We exclusively focus on those CLDs that perform power control with the goal of suppressing MAI. CLDs that primarily aim at energy savings or topology control are not taken into account. Furthermore, we do not incorporate approaches that rely on a central entity.

We start the summary with approaches that exchange information, e.g., tables, between different participants, in order to inform nodes about power information between neighbors (so-called power-exchange) [2], [12], to gain routing information for multiple different power levels [13], to get interference tolerance levels of the neighborhood [14], or to achieve information about link gains between two neighboring nodes (indirect links) [15]. Due to the prohibitive overhead expected for timevarying channels, these approaches are solely applicable in non-fading environments. This is explicitly formulated as a constraint in [16]. For the proposed distributed power-control algorithm with active link protection (DPC/ALP) the authors restrict the application field to quasi-static channels where the time scale of mobility is much larger than that of power adaptation.

Other approaches use a separate control channel besides the data channel, to inform transmitters in their vicinity on the additional amount of interference they can tolerate [17], or to transmit all control signaling separately to avoid collisions between control and data packets [18]. Using a separate control channel requires additional resources, dependent on the amount of control information exchanged. Also, though it avoids collisions of incoming control and data messages, it does not automatically assure that control messages from different nodes do not collide. Furthermore, if data and control signals are transmitted at the same time, a node is either required to own two transceivers, as assumed in [15], [17], [18], in order to simultaneously receive and transmit that is either very costly in terms of hardware; or it is deaf to all incoming signaling on the control channel while it is transmitting data, leading to well known performance degradations due to deafness. Transmitting data and control signals as a solution in a 
time-division manner as argued by the authors of [19] to avoid two transceivers, however, makes the application of a separate control channel unnecessary.

As discussed so far, most approaches rely on impractical assumptions such as additional hardware or time invariant channels. Only a few proposals [1], [3], [20], which we discuss in the following, seem to be designed without such strict assumptions and may be applicable in practical scenarios.

In [1] the asynchronous POWMAC protocol is proposed. This protocol uses a so-called access window phase, to agree on a set of transmissions that can simultaneously proceed. During the signaling for a transmission, each potential receiver announces the transmission power to be used by the communication partner as well as a common maximum interference level it can tolerate from a single newly starting transmission. Each transmitter that starts its own signaling afterwards must assure that it does not violate any of the interference tolerance levels included in preceding signals. After the access window phase multiple data transmissions can take place simultaneously.

Based on the POWMAC protocol, the so-called adaptive transmission power control protocol (ATPMAC) [20] was developed. The authors of [20] avoid reserving time for the access window phase by transmitting control signaling in parallel to data transmissions.

The major drawback of both schemes [1], [20] is the assumption of one common maximum interference level that is the same for all interfering nodes. This level is more or less the overall tolerable interference power at a receiver divided by the number of interferers in its vicinity. However, defining one common average interference level is highly inefficient, since the interference strongly varies with the distance (or channel) between the interferer and the interfered node. While a distant transmitter is allowed to cause more interference than it actually requires due to the common interference level, a nearby node might fail to hold the common interference limit and thus abstain from transmitting.

Due to the shortcomings of the algorithms presented be-forehand [1], [20], the so-called PBOA [3] is chosen as the most reasonable reference scheme. We will present it in the following.

\section{PBOA}

The PBOA protocol assumes a certain time slotted structure, called frame that is depicted in Figure 1. The first part of the frame is related to a contention phase and consists of several pairs of minislots. Each minislot is divided into the transmission of an RTS and a CTS signal. The second part of the frame is used for the transmission of data. Notice that no additional acknowledgment is assumed by the authors of [3]. Before the

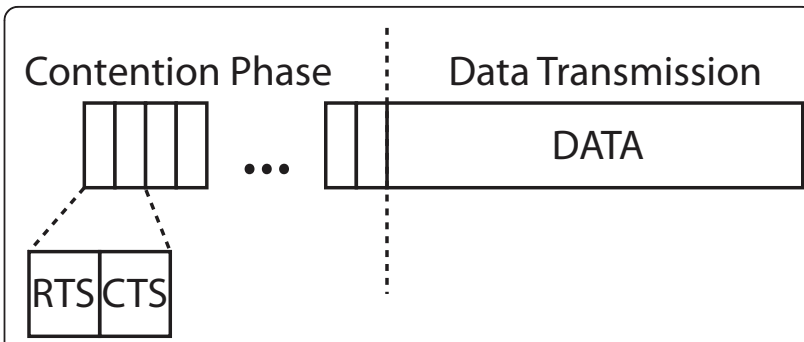

Figure 1 General frame structure of the PBOA

data is transmitted, the different terminals, willing to transmit, start contending for channel access, i.e., at the beginning of the contention phase each potential transmitter simultaneously transmits its RTS signal with maximum power. Figure 2 illustrates this (first minislot). $\mathrm{T}_{1}$ to $\mathrm{T}_{4}$ thereby represent simultaneous transmissions during the contention phase.

If the intended receiver can decode the RTS, it replies with a CTS, also with maximum power. Depending on its receive signal-to-interference-and-noise-ratio (SINR) and its actual SINR requirement, it includes a factor into the CTS that tells its associated transmitter how much to power down in the next RTS minislot of the contention phase.

An exemplary behavior is depicted in Figure 2 in the middle (second minislot), where the transmission power of $\mathrm{T}_{4}$ starts to decrease. The successive power reduction goes on in consecutive minislots, unless a minimum for the acceptable transmission power is reached. Afterwards, the receiver of $\mathrm{T}_{4}$ will abstain from transmitting further CTS messages. Its associated transmitter, however, will proceed transmitting RTS signals with the minimum transmission power until the contention phase ends. This enables other receivers to still correctly estimate the interference expected during data transmission.

If a transmitter does not receive a CTS during one minislot, it will stay contending during the consecutive slot with a so-called win probability $p$, or it will go to backoff and turn into a potential receiving node until the end of the frame with the probability of $1-p$.

In Figure $2 \mathrm{~T}_{1}, \mathrm{~T}_{2}$ and $\mathrm{T}_{3}$ are not successful during the first minislot of the contention phase. While $\mathrm{T}_{2}$ looses and goes into backoff, $\mathrm{T}_{1}$ and $\mathrm{T}_{3}$ try to succeed again during the second minislot. Notice, however, that $\mathrm{T}_{3}$ chooses a different receiver, namely the receiver of the second packet in its transmission queue. This is proposed by the authors of PBOA, in order to increase the probability that RTS messages reach the intended receivers.

By progressively reducing transmission powers and the number of potential transmitters (backoff), other transmitters are given more chance to reach their intended 


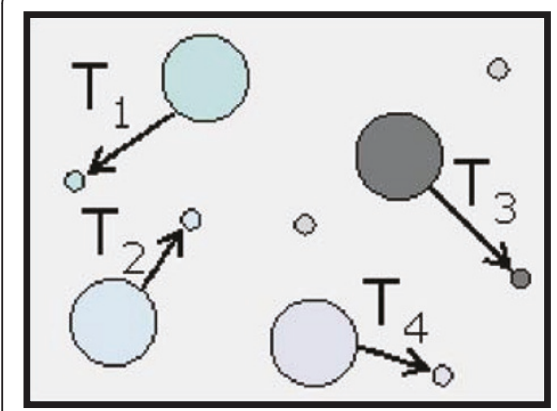

First Minislot

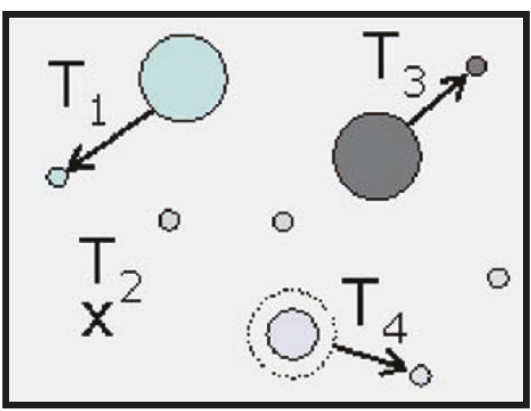

Second Minislot

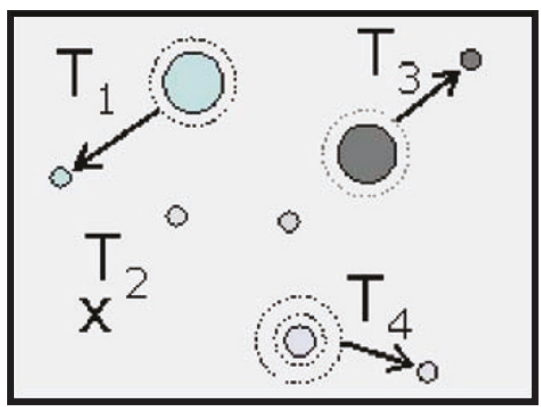

Third Minislot

Figure 2 Power adaptation and backoff during the contention phase of the PBOA protocol.

receivers. This is illustrated in Figure 2 in the third minislot, where $T_{1}$ and $T_{3}$ can reach their respective receivers due to the reduced interference.

After the contention phase all successful transmitters send their data to their intended receivers with the minimum transmission power they agreed on. The authors claim that an additional acknowledge is not required, since the channel is assumed to stay constant for the duration of the whole frame and thus the transmission must be successful [3].

\section{MUD-based medium access}

\section{A. MUD functional principle}

In contrast to power control at the transmitter, the principle of MUD is to deal with interference at the receiver. The principle is that the receiver detects not only the desired signal but also the interference that is subtracted from the observation signal to have a better estimate of the desired signal. This process can be repeated until the error performance becomes satisfactory. The iterative MUD structure at the receiver is illustrated in Figure 3. The number of decoder branches $K$ ' thereby determines the capability of canceling interferences as well as the complexity of the receiver. The multiuser detector attempts to cancel the interferences by making use of the estimates from the decoders. This is called soft interference cancelation:

$$
\tilde{y}_{i}^{(k)}=y_{i}-\sum_{k \prime-1, k^{\prime} \neq k}^{K^{\prime}} \hat{h}^{\left(k^{\prime}\right)} \tilde{s}_{i}^{\left(k^{\prime}\right)},
$$

where $\tilde{s}_{i}^{(k)}$ is the symbol replica computed from the input from the decoder. The channels for $K^{\prime}$ transmitting nodes have to be estimated as $\hat{h}^{(k)}$. It should be emphasized that not only the channels for $K$ ' users have to be estimated, but also the user-distinct signatures (e. g., spreading sequences for DS-CDMA) for $K^{\prime}$ users have to be known at the receiver to perform the MUD as seen from Figure 3. The observation signal after the soft interference cancelation in (1) can be utilized for computing the improved estimate of the desired signal as well as interference, which are then sent to the decoder. This process is iteratively performed until the estimate of the desired signal is sufficiently improved. Interferences are eventually discarded.

\section{B. Overview of MUD-based CLDs}

We proceed with a State-of-the-Art of MUD-based CLDs for wireless ad hoc networks. We start with a major challenge MUD faces in wireless ad hoc networks and categorize the algorithms dependent on their assumptions and solutions to this challenge.

As already stated in Sect. IV-A MUD requires channel state information on the receiver side, i.e., in order to successfully cancel a stream sent by a transmitter, the receiver must estimate the channel from this transmitter beforehand. In fading environments, the estimation is only valid during a limited time period, the so-called coherence time. This is the time span during which the channel is assumed to stay fairly constant.

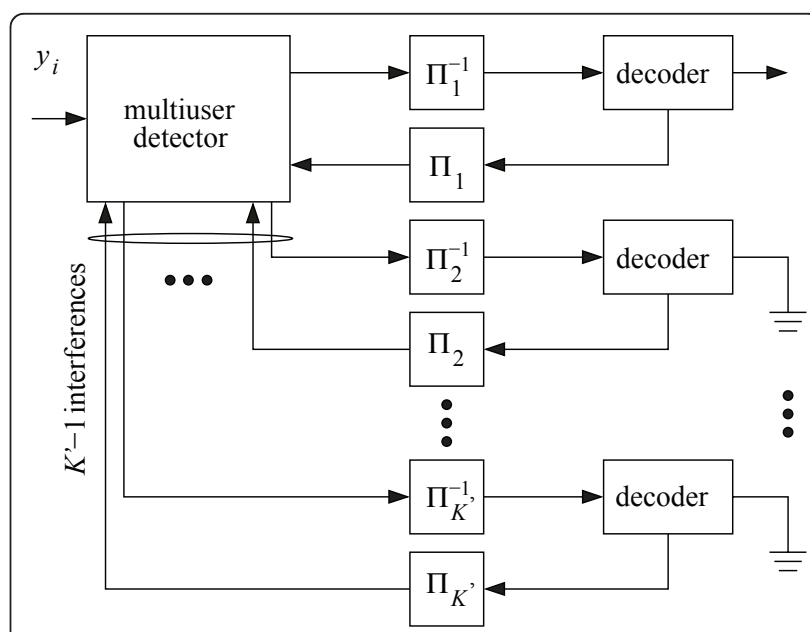

Figure 3 Iterative receiver structure with $K^{\prime}$ decoder branches. 
Notice that channel estimation may not be performed reliably if multiple transmitters simultaneously transmit the pilots, since the signals of all transmitters superimpose, unless the pilots are somehow made orthogonal, i. e., by individual orthogonal codes.

We start with two approaches, namely [8] and [21] that both address Multiple Input Multiple Output (MIMO)-based CLDs with spatial multiplexing on the transmitter side and a V-BLAST type multiuser detector on the receiver side. The approaches adapt the 802.11 CSMA/CA scheme in the sense that the RTS/CTS handshake is not applied to avoid collisions but rather to agree on multiple parallel transmissions. Both approaches offer interesting insights and strategies with respect to MUD in ad hoc networks. However, both assume that nodes are frame level synchronized and all nodes willing to transmit simultaneously transmit their RTS signals. Thus, all nodes in the network may have to transmit pilots beforehand in, e.g., a time division manner with all their antennas to assure that channel state information is provided to separate signals during the control signaling phase.

Such a channel estimation phase is proposed in [22]. The authors claim that this requires only a short period of time. However, for high node densities in fully connected networks this phase is expected to cause prohibitive overhead.

Zhang et al. [23] present a MAC protocol design that combines CDMA with a MUD receiver. In order to achieve a distributed priority based neighborhood scheduling, the authors propose to separate the nodes into groups. Each group simultaneously transmits their RTS information within one RTS slot. By repeating overheard messages by members of other groups in consecutive RTS slots the authors distribute the information about priorities and planned transmissions in the whole network.

The authors assume that each node has an individual code assigned what makes the reception of multiple parallel RTS signals in principle possible. This is, however, a very bandwidth demanding assumption, since for a large number of users the spreading sequences have noticeable length.

The authors of [24] exactly address this issue and assume for their algorithm as a prerequisite that the neighbor density is limited such that channel estimation and decoding is possible. They assume that each node has one individual code out of a code list that is common and known to all nodes in the network. Under these assumptions, the authors propose a distributed scheduling algorithm that exploits multiuser and spatial diversity gains by selecting nodes and antennas with good channel conditions.

In order to overcome limitations regarding the node density due to channel estimation requirements, and also to avoid that nodes having a smaller number of antennas than other nodes or even only a single antenna are starved, a possible solution is to avoid MUD as a prerequisite during the control signaling phase. This is partly suggested by the authors of [7].

For their Interference Division Multiple Access protocol they assume synchrony on a frame level basis. A frame thereby consists of an RTS zone, a CTS zone, a DATA zone, and an acknowledgment (ACK) zone and is consecutively repeated over time. Instead of allowing all nodes to simultaneously transmit their signals during the RTS and CTS zone, which would lead to the disadvantages summarized beforehand, the authors subdivide these zones into multiple RTS and CTS slots. Thus, the authors can offer channel state information for the transmissions, since all RTS signals are transmitted in a TDMA manner. However, still all ACK signals are simultaneously transmitted, requiring multiuser capabilities for their successful reception.

All challenges summarized beforehand are overcome by the so-called MUD-MAC protocol [9]. This protocol gains the channel information required for the multiuser detector in a fully distributed way and it also supports nodes that are equipped with a smaller number of MUD branches or even no multiuser detection capabilities. This is achieved since the detection of the control signaling does not require MUD as a mandatory capability. Hence, we choose this protocol as a reference scheme and summarize it in the following.

\section{The MUD-MAC Protocol}

Similar to PBOA, MUD-MAC requires a time-slotted structure, referred to as block. Each data frame is subdivided into $N$ blocks. The block structure of MUD-MAC is depicted in Figure 4.

Each block consists of several control signals, namely announcement (ANN), objection (OBJ), and acknowledgment (ACK), and a slot for data transmission (DATA). Notice that during the control signaling slots, no MUD capabilities are required.

Unlike PBOA, transmitters should not start their control signaling simultaneously. Instead, each transmitter randomly chooses one minislot and abstains from a planned transmission if it senses another transmitter signaling in an earlier slot. This kind of contention resolution mostly avoids collisions during the ANN phase.

\begin{tabular}{|c|c|c|c|c|}
\hline \multicolumn{5}{|c|}{ minislots } \\
\hline$\ldots$ & ANN & OBJ & DATA & ACK \\
\hline \multicolumn{3}{|c|}{ No MUD } & MUD & No MUD \\
\hline \multicolumn{5}{|c|}{ Fiqure 4 One block of the MUD-MAC protocol } \\
\hline
\end{tabular}


The successful transmitter announces the planned transmission to its associated receiver. It includes a signature used during the data phase into the ANN signal. This signature is required, since a spread spectrum multiple access scheme, e.g., CDMA or IDMA, is considered. Notice, however, that the spreading code does not need to be able to separate all users in the whole network. Thus, a moderate spreading (e.g., 11) can be applied. A transmission lasting $N$ blocks is announced only once per packet. A new transmission can be started in each new ANN slot, resulting in a maximum of $N$ parallel transmissions.

With the help of the ANN signals, channel estimation can be performed at the associated receiver as well as at receivers that are already involved in ongoing transmissions. During the OBJ phase, the latter ones have the opportunity to object to the planned transmission. This happens, if they cannot handle the additional interference, e.g., if they have no more free MUD branches.

If no OBJ can be sensed, the transmitter starts transmitting the first of $N$ blocks. The size of the blocks is thereby chosen such that the channel coherence time is larger than the time required for the transmission of all $N$ blocks. If the transmission is successful, the receiver acknowledges the reception of multiple blocks once at the end of the transmission. Since transmissions start one after the other and last for $N$ blocks, only one ACK will be proceeded in one slot.

\section{How to provide a fair comparison}

In this section we explain some adjustments of different assumptions that we performed to achieve a fair comparison between the two reference schemes and 802.11.

\section{A. Network layer assumptions}

In the PBOA protocol, the authors assume that transmitters can switch to the next receiver awaiting the transmission of a packet in their queue, in case a transmitter is not successful during an RTS slot, as it is the case for $\mathrm{T}_{3}$ between first and second minislot in Figure 2. In order to be fair to MUD-MAC and 802.11, we stick to a pure First In First Out (FIFO) packet queueing for all schemes instead.

\section{B. MAC layer assumptions}

802.11 and MUD-MAC originally assume a globally unique address space for the nodes, resulting in 6 bytes per node ID. Since PBOA includes the node ID into each of the RTS/CTS minislots, a global address would result in prohibitive over-head. Also, it is not commonly required to share a global unique address space in ad hoc networks since the number of active nodes is rather limited. Thus, a locally unique address space of 1 byte is assumed for all schemes and the MAC overhead is accordingly adapted. The MAC overhead for the two CLDs includes all overhead contained in the 802.11 MAC header. Only the bits for transmission durations are not required for the two CLDs, since they are frame-level synchronous and thus the transmission duration is fixed.

We assume a frame length of 8192 information bits for all schemes. For the MUD-MAC CLD, a packet of 8192 bits is split into $N=4$ data blocks.

\section{Physical layer assumptions}

The PHY overhead for both CLDs includes all bits from the PHY header of 802.11 except the ones that the asynchronous 802.11 protocol requires for synchronization, since PBOA and MUD-MAC are frame-level synchronous.

The authors of the PBOA protocol assume a so-called Brickwall model, i.e., if the SINR of a packet is lower than a certain minimum SINR, the packet is lost, if it is higher, the packet is received error free. During the power adaptation phase, all nodes assume this Brickwall-SINR as the minimum SINR required. Besides the fact that this kind of model simplifies reality, it is stricter than a model that estimates a Packet Error Rate (PER) dependent on the SINR and looses the packets with this probability. This is assumed for the 802.11 and the MUD-MAC protocol. Thus, in order to avoid disadvantaging of the PBOA protocol, the physical layer is assumed to loose packets dependent on a PER for all schemes. The power adaptation during the contention phase of the PBOA protocol thereby assumes a minimum receive SINR of $14 \mathrm{~dB}$, corresponding to a packet error probability of $10^{-2}$.

For the MUD-MAC protocol, a moderate spreading with spreading gain 11 is assumed, resulting in an 11times increase of bandwidth. In the $802.11 \mathrm{~b}$ protocol, the same spreading gain of 11 is applied against out-ofband interferers. However, PBOA assumes only a single band transmission and is thus naturally penalized by the comparison. Thus, in order to balance the bandwidth requirements for all schemes, we assume that $\mathrm{PBOA}$ also performs some kind of spreading and include a spreading gain of 11 during the interference calculations for PBOA. Receivers already include this spreading gain while they estimate how much their associated partner can power down.

\section{Energy efficiency}

Since PBOA avoids interference by individually reducing transmit power levels, besides an increased spatial reuse, also the energy efficiency can be improved. We do, however, not compare the schemes regarding the energy efficiency, since the MUD-MAC protocol is not designed to additionally achieve energy savings. 
Reducing the transmit power level such that it appropriately serves the receiver depends on the underlying modulation and coding scheme and is out of scope of this article. It seems, however, to be a straight forward improvement for the MUD-MAC protocol in the future.

\section{QoS parameters}

In order to get insight into the QoS offered by a CLD, in addition to the system throughput, delay and fairness have to be carefully investigated. We describe the parameters that we apply to measure the achieved QoS in the sequel.

\section{A. Throughput}

We investigate the aggregate throughput offered by the comparison schemes. The aggregate throughput thereby accounts for the sum of information bits of all packets successfully received by all nodes in the network during the simulation time of $12 \mathrm{~s}$, averaged over this simulation time. The simulation time equates to about 8000 contention cycles, what seems to be sufficient to achieve valid data statistics also about the long term behavior of the protocols. One run of $12 \mathrm{~s}$ is repeated 40 times while every time the nodes are newly randomly placed for each investigated offered traffic load and subsequently averaged to approximate the mean value. Investigations with the $95 \%$ confidence interval showed that 40 iterations are sufficient. All following measures are also averaged over the simulation time of $12 \mathrm{~s}$ and 40 realizations.

Opposite to the aggregate throughput, for the throughput per node the information bits successfully transmitted within the simulation time are not summed up over all nodes in the network, but only per node and subsequently averaged.

\section{B. Delay}

We measure the delay as the delay per packet that nodes experience while transmitting. According to [25], besides traffic that has no delay restrictions, there exist real-time streaming services with very strict delay requirements $(150 \mathrm{~ms}-250 \mathrm{~ms})$ and non-real time services that are interactive. The latter require at least delays that are lower than $2 \mathrm{~s}$. However, for, e.g., web browsing, as service contained in this group, a maximum delay of 0.5 seconds would be desirable [25]. Thus, we restrict the maximum delay $\Delta_{\max }$ a packet can tolerate to $1 \mathrm{~s}$. If the delay exceeds this limit, the packet is removed from the packet queue and lost.

We define the mean packet delay $\overline{\Delta_{\mathrm{p} k}}$ of the received packets each node $k$ experiences as the sum of the packet delays $\Delta_{\mathrm{p} k, i}$ of all successfully transmitted packets $i$ over the number of successfully transmitted packets $N_{k}$ for this node, respectively:

$$
\overline{\Delta_{\mathrm{p} k}}=\frac{\sum_{i=1}^{N_{k}} \Delta_{\mathrm{p} k, i}}{N_{k}} .
$$

In order to take fairness into consideration as well, we subsequently evaluate the median of these mean packet delays per node. Unlike a mean, the median is insensible to outliers. It is the value separating the higher half of the realizations from the lower half. In case of unfair medium access, single nodes that are frequently granted medium access can significantly decrease the overall mean delay. However, the median will not be strongly influenced by these nodes.

\section{Fairness}

In order to get insight into the fairness behavior of the CLDs, we evaluate the variance of both, the mean packet delay values $\overline{\Delta_{\mathrm{p} k}}$ for different nodes, and the one for the average throughput per node. It can be stated that the lower the variance of these values is, the fairer is the access to the medium.

Another measure for fairness of medium access is the so-called Jain's fairness index [26]. This index is defined for $K$ nodes as

$$
F_{\mathrm{J}}(w)=\frac{\left(\sum_{k=1}^{K} g_{k}(w)\right)^{2}}{K \sum_{k=1}^{K} g_{k}^{2}(w)} \text { with } 0<F_{\mathrm{J}}(w) \leq 1
$$

where $w$ reflects a sliding window with a size of multiple packets, and $g_{k}(w)$ reflects the fraction of the overall medium access, a node $k$ achieved within this window. The window is stepwise increased over the pattern of medium accesses, thereby reflecting the change from short-term to long-term fairness.

In case of perfectly fair channel access, all $g_{k}(w)$ equal $\frac{1}{K}$ and Jain's fairness index is equal to 1 . A scheme is fairer if its Jain's fairness index is closer to 1 and vice versa.

\section{Simulation results}

The following section presents simulation results that compare the QoS offered by PBOA and MUD-MAC measured in terms of aggregate throughput, delay, and fairness. Additionally, we compare the two CLDs to the 802.11 protocol.

The system parameters are listed in Table 1. The number of minislots assumed is a design parameter, as also discussed in [3]. For the PBOA-MAC protocol, it furthermore is strongly related to the win probability $p$. Thus, regarding the number of minislots, we stick to the proposal in [3] and adapt the win probability $p$ instead. We use a win probability $p=0.7$, since this value resulted in the best performance in our simulations. 
Table 1 Simulation parameters

\begin{tabular}{|c|c|c|c|}
\hline & MUD-MAC & PBOA & 802.11 \\
\hline Control sig. bit rate & $1 \mathrm{Mbit} / \mathrm{s}$ & & \\
\hline Data bit rate & $2 \mathrm{Mbit} / \mathrm{s}$ & & \\
\hline Packet size & 8192 bit & & \\
\hline Number of minislots & 10 & 15 & - \\
\hline Transmission Power & $100 \mathrm{~mW}$ & & \\
\hline Decoding sensitivity & $-81 \mathrm{dBm}$ & & \\
\hline Carrier sensing sensitivity & $-91 \mathrm{dBm}$ & & \\
\hline Carrier frequency & $2 \mathrm{GHz}$ & & \\
\hline Bandwidth & $22 \mathrm{MHz}$ & & \\
\hline Path loss exponent & 3 & & \\
\hline Modulation scheme data & QPSK & & \\
\hline Modulation scheme control signals & BPSK & & \\
\hline
\end{tabular}

For the MUD-MAC protocol, we choose the number of minislots such that is balances losses due to increased over-head in a medium traffic load scenario with packet looses due to control message collisions in a high traffic load scenario. Notice that we do not assume that the number of minislots can be adapted dependent on the traffic load in the scenario for either of the schemes.

We assume Poisson packet arrivals, such that the inter-arrival times of the packets are exponentially distributed. The channel is modeled with a modified free space path loss model, and line-of-sight is assumed. Fading is not considered in the channel model. Since the duration of a frame ( $N$ consecutive blocks) of MUDMAC as well as the frame duration of PBOA are similar and both assume that the channel stays constant for the transmission of the complete frame, we do not expect that the results of the comparison are strongly influenced by this. Including a block-fading channel model is expected to reduce the performance of both schemes, MUD-MAC as well as PBOA, in a similar way.

We model the probability that a packet is corrupted according to the error probability of the additive white gaussian noise channel [27]. As modulation alphabet, we assume BPSK for the control packets, and QPSK for the data transmissions. For a more detailed description of the channel model, please refer to [9].

For the MUD-MAC protocol, we simulate a MUD receiver with four decoder branches, since this seem to be a reasonable assumption with respect to the computational complexity of the MUD detector. Furthermore, also a low complexity receiver with two decoder branches is simulated.

In order to, on the one hand, get insight into the scaling behavior of the MAC protocols regarding increasing node numbers and, on the other hand, still achieve acceptable simulation times, we choose the overall number of nodes to be simulated to 50 . At the beginning of the simulation each node randomly chooses one other node out of the set of nodes within communication range as a sink. Notice that we assume all 50 nodes to be active, i.e., all nodes generate packets and potentially transmit during simulation time. We refer to the expression offered traffic as the sum of packets generated at all nodes during simulation time in the following.

\section{A. Throughput comparison}

We start our investigations regarding the QoS by comparing the aggregate throughput achieved by both schemes. In order to investigate the applicability of the CLDs in different environments, we simulate two scenarios with strongly varying interference conditions:

(1) Partly connected network: The area investigated is $500 \mathrm{~m} \times 500 \mathrm{~m}$. Not all terminals are within the communication range of each other. Here an appropriate MAC layer design is expected to be able to achieve good gains in terms of spatial reuse.

(2) Fully connected network: The network area is 50 $\mathrm{m} \times 50 \mathrm{~m}$. Interference is high, since each terminal is within the communication range of all other terminals. Here, the contention is expected to be too severe to result in spatial reuse by an appropriate MAC layer design alone. This scenario offers insight into the capability of the underlying physical layer, to handle interference situations that would lead to a TDMA kind of contention resolution with no spatial reuse by a pure MAC layer design.

We do not simulate specific topologies like star or line setup, since nodes in an ad hoc networks are usually randomly distributed without specific topologies.

We place the 50 nodes uniformly in both scenarios. Figure 5 shows the aggregate throughput over the offered traffic for the partly connected network. Both CLDs offer gains over the 802.11 protocol, since they allow for spatial reuse while the CSMA/CA algorithm of 802.11 blocks all transmissions except one within mutual sensing range. This results in an aggregate throughput of $7.52 \mathrm{Mbit} / \mathrm{s}$ for the MUD-MAC protocol with four branches (7.46 Mbit/s with two branches), $5.39 \mathrm{Mbit} / \mathrm{s}$ for the PBOA protocol and only 4.07 Mbit/ $\mathrm{s}$ for the 802.11 protocol if the offered traffic is 9.5 Mbit/s.

In the fully connected network (Figure 6), the situation is different. Still MUD-MAC with four branches (3.81 Mbit/s throughput at $6 \mathrm{Mbit} / \mathrm{s}$ ) as well as two branches $(2.22 \mathrm{Mbit} / \mathrm{s}$ throughput at $6 \mathrm{Mbit} / \mathrm{s}) \mathrm{can}$ remarkably outperform 802.11 (1.36 Mbit/s throughput at $6 \mathrm{Mbit} / \mathrm{s})$. However, the power control-based PBOA protocol $(1.33 \mathrm{Mbit} / \mathrm{s}$ throughput at $6 \mathrm{Mbit} / \mathrm{s})$ can not significantly gain compared to 802.11 and at $6 \mathrm{Mbit} / \mathrm{s}$ 


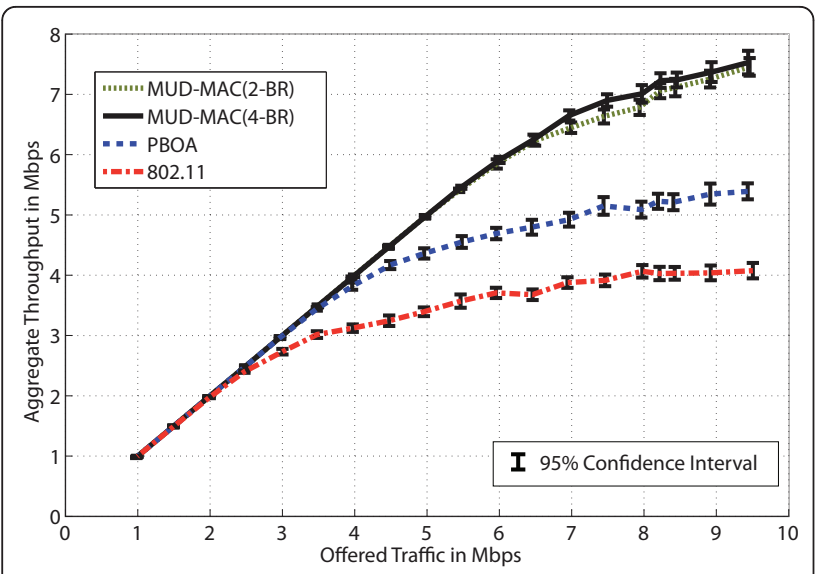

Figure 5 Overall throughput versus offered traffic in a random

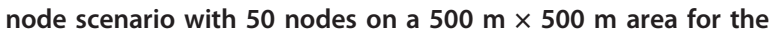
three MAC protocols.

gets even slightly worse than 802.11. An overview of the resulting additional throughput gains in percentage for the cross-layer solutions compared to 802.11 at 9.5 Mbit/s respective $6 \mathrm{Mbit} / \mathrm{s}$ is given in Table 2 .

In order to explain these results, we have a closer look into the contention phase of PBOA, depicted in Figure 7 . In the lower row, the 1 st, 4 th, 7 th, and 15 th minislot of an exemplary contention phase in a $500 \mathrm{~m} \times 500 \mathrm{~m}$ partly connected network are depicted. During the RTS phase of the 1st minislot, all nodes simultaneously transmit their RTS signals. Blue connecting lines between the individual nodes indicate that these nodes are within mutual communication range $(\approx 126 \mathrm{~m})$. During the 4 th minislot one receiving node, marked with a yellow circle, was successful in decoding an RTS signal and now replies with a CTS signal. Still a noticeable number of nodes is awaiting CTS response. The node replying has

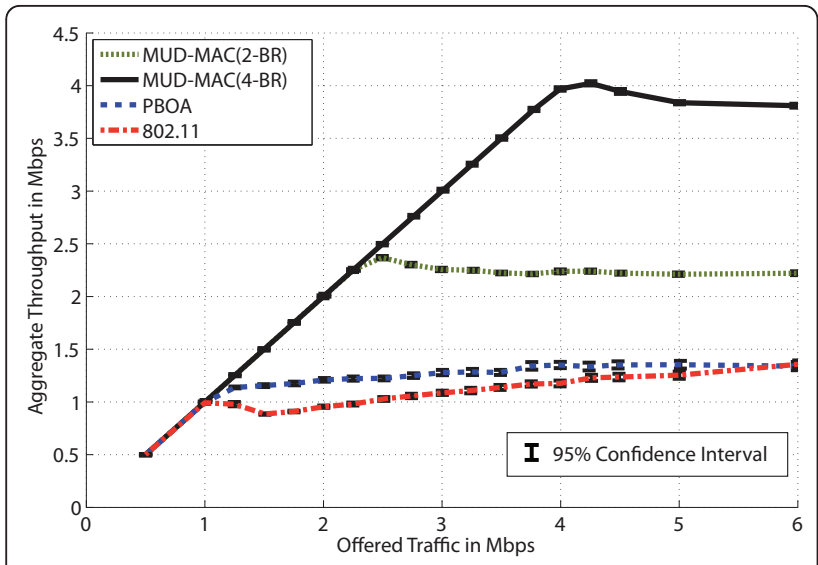

Figure 6 Overall throughput versus offered traffic in a random node scenario with 50 nodes on a $50 \mathrm{~m} \times 50 \mathrm{~m}$ area for the three MAC protocols.
Table 2 Throughput gains in percent compared to $\mathbf{8 0 2 . 1 1}$

\begin{tabular}{cll}
\hline & Figure 5-500 $\mathbf{~ m}$ & Figure 6-50 $\mathbf{~}$ \\
\hline PBOA & 32.4 & -2.2 \\
MUD-MAC (2-BR) & 83.3 & 63.2 \\
MUD-MAC (4-BR) & 84.7 & 180.1 \\
\hline
\end{tabular}

advantages compared to other nodes in the scenario regarding the decoding of the RTS signal, since it is not in the middle of the scenario, where many nodes are within mutual communication range, but at a border and also the majority of its neighbors already gave up transmitting RTS signals. Additionally, its associated partner is very close.

Similar properties can be observed during the 7 th minislot. There, the number of nodes replying with a CTS is increased to 3 . All receivers have in common that they are very close to their associated partners. Also, in their communication vicinity, no other active nodes can be found. In the 15th minislot the number of nodes replying with CTS signals is increased to five. Notice, however, that the number of simultaneous transmissions that will take place during the subsequent data phase is, however, still four, since one node, node 31 , marked with a rectangle, replies without an associated partner. This was caused by a CTS packet loss, resulting in the unsuccessful partner backing off.

What can be seen from this behavior is that most of the parallel transmissions only became possible, since the concurrent transmissions in the communication vicinity backed of and the associated partners are close.

Opposite to power control-based CLD, besides a larger amount of parallel transmissions compared to PBOA, for MUD-MAC also some transmissions take place in close vicinity and partners do not necessitate to be close, as depicted on the right hand side of Figure 8. There an exemplary data transmission is depicted for MUD-MAC in the partly connected network. The contention resolution of the MUD-MAC protocol does not block all but one transmissions within mutual communication range, which is mostly the case for the power control-based CLD. Instead, for the MUD-MAC protocol the physical and MAC layer interact and thus provide a higher spatial reuse, as was also shown in [10].

These observations get even stronger supported, if the contention phase of the fully connected network is further investigated. The upper row of Figure 7 shows the 1st, 4th, 7th, and 15th minislot of an exemplary contention phase in the fully connected network for the PBOA-MAC protocol. Similar to the $500 \mathrm{~m}$ scenario, during the 1st minislot all potential transmitters simultaneously transmit their RTS signals. This time, however, the blue lines representing node pairs within communication range are very dense compared to the 


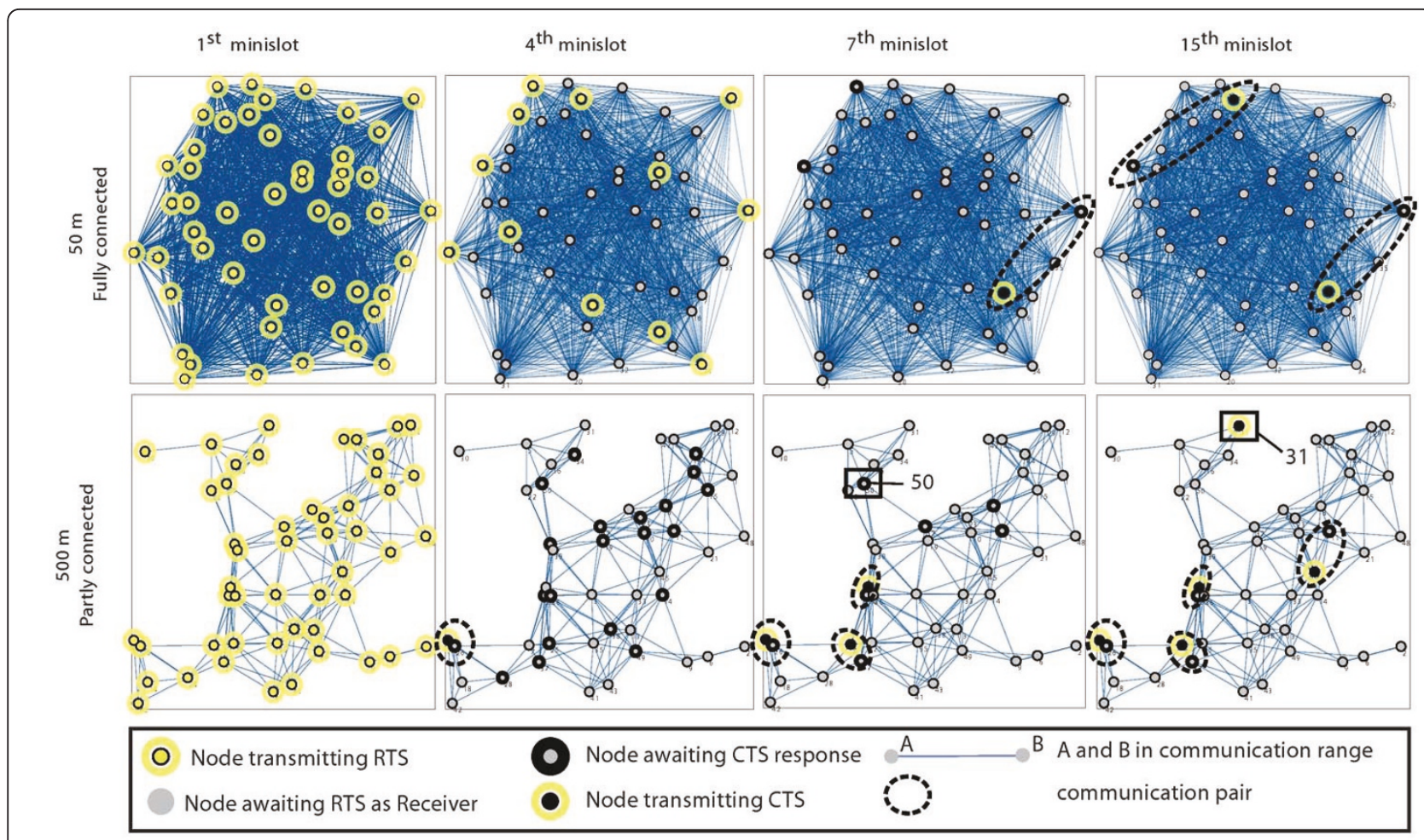

Figure 7 Node states during the 1st, 4th, 7th, and 15th minislot of the contention phase of the PBOA protocol for the fully connected network (upper row) and the partly connected network (lower row)

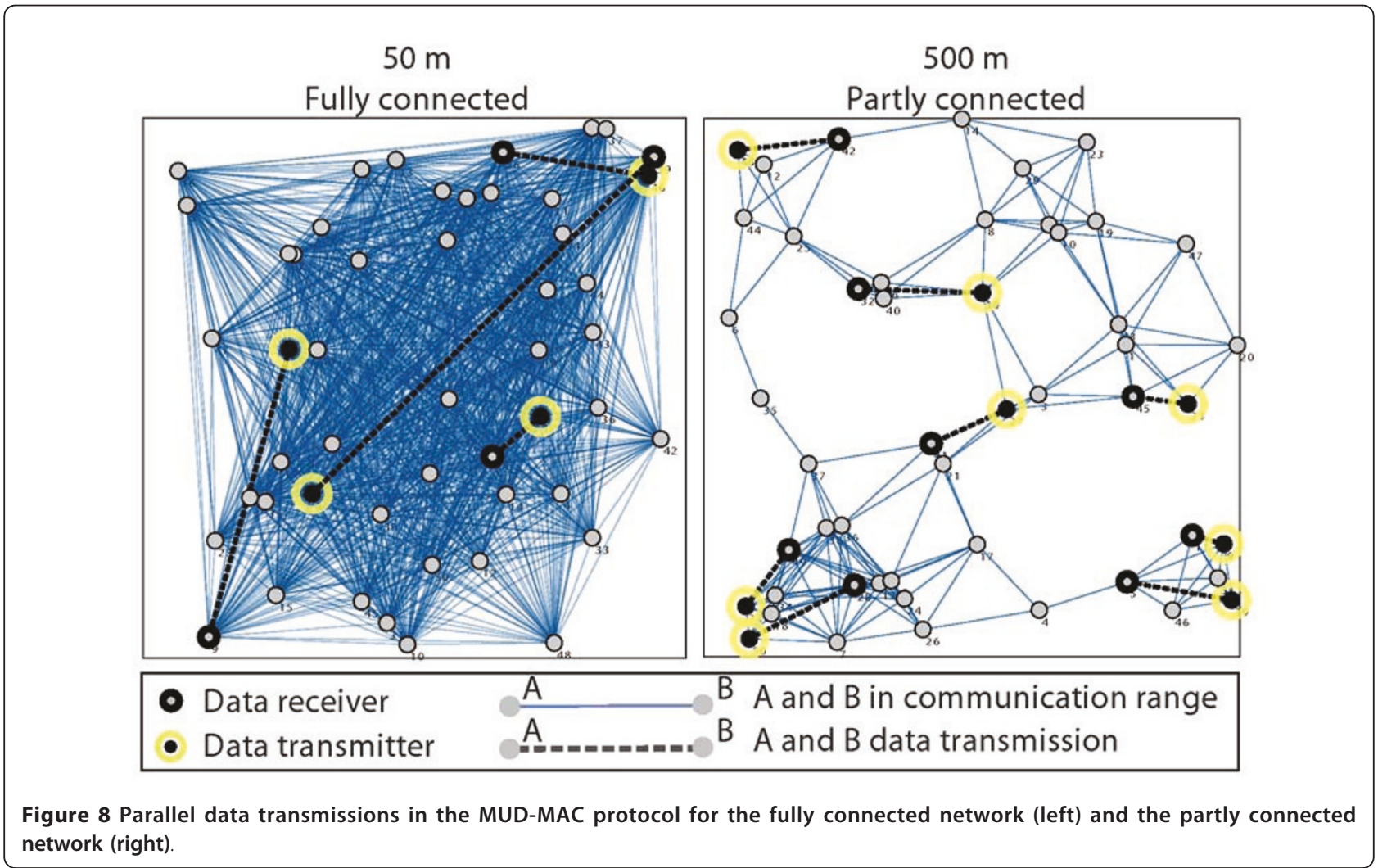


$500 \mathrm{~m}$ scenario, thus reflecting the strongly declined interference situation. This interference situation cannot be handled by means of power control which is also shown by the contention situation of the 4th minislot. Opposite to the $500 \mathrm{~m}$ scenario, not the CTS phase but the RTS phase is depicted since still no CTS is started and thus in the event-driven simulation environment no visualization output is produced. During the associated RTS, however, it can already be seen that many nodes willing to transmit during the 1 st minislot already backed off, since they were not successful. During the CTS phase of the 7th minislot, one node managed to decode an RTS signal and replies with a CTS to its partner. After 15 minislots, two communication pairs remain. Notice that they are so far apart that the interference level is much lower than the receive signal level.

Opposite to the observations presented beforehand, the MUD-based MUD-MAC can not only handle the interference situation, but even increase the spatial reuse in the high traffic scenario by fully exploiting all four decoder branches. This is shown by comparing the left and the right hand side of Figure 8 . While in the right part for the $500 \mathrm{~m}$ scenario never more than two parallel transmissions are within communication range which is mostly the case in the medium density scenario, in the left part for the $50 \mathrm{~m}$ scenario all four branches are exploited, leading to four parallel transmissions. From the above presented results it can be observed that power control-based CLDs can only be applied in medium density interference situations, whereas MUD in interaction with an appropriate MAC layer is also applicable if the interference situation gets severe. The gain achievable scales thereby in high interference situations with the number of decoder branches, but also low-complexity two-branch MUD receiver can handle the severe interference situation cp. Figure 6. Since power control-based CLD cannot be applied in severe interference situations, we restrict all further performed simulations to the medium node density scenario.

\section{B. Delay and fairness in the random topology}

After we compared the two CLDs and 802.11 in terms of throughput, we now compare delay and fairness. We start our investigations with the median of the mean packet delays $\overline{\Delta_{\mathrm{p} k}}$ of all nodes over the aggregate delivered traffic, depicted in Figure 9.

MUD-MAC with both, two and four branches, clearly outperforms the PBOA CLD. In order to investigate the performance further, we look at real-time streaming services with very high delay requirements of $200 \mathrm{~ms}$ (150 ms-250 ms [25])-marked with a dashed line in Figure 9. Even here, the MUD-based CLD offers about $6.5 \mathrm{Mbit} / \mathrm{s}$

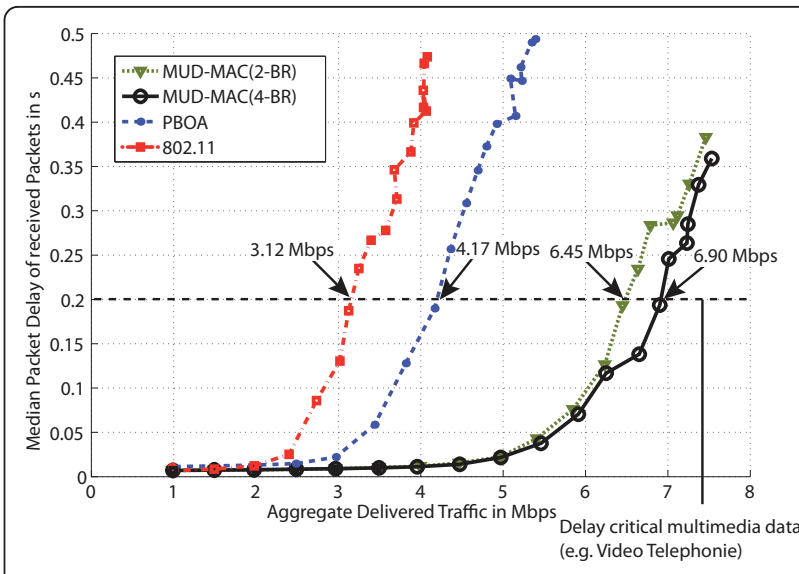

Figure 9 Median of mean packet delay $\overline{\Delta_{\mathrm{p} k}}$ over delivered traffic for the 802.11, the MUD-MAC and the PBOA protocol with 50 nodes in a $500 \mathrm{~m} \times 500 \mathrm{~m}$ random network.

(6.90 Mbit/s with four branches, $6.45 \mathrm{Mbit} / \mathrm{s}$ with two branches) delivered traffic. This corresponds to a gain of $121 \%$ (four branches), and 107\% (two branches), over 802.11 (3.12 Mbit/s).

The power control-based cross-layer solution cannot offer such a throughput improvement for applications with that stringent delay requirements. However, with 4.17 Mbit/s aggregate delivered traffic it can achieve a gain of $33.6 \%$ over the 802.11 protocol. Still, MUDMAC with four branches provides $65.5 \%$ more throughput for highly time critical applications than PBOA.

To get insight into the fairness of the CLDs, the variance of the throughput per node over the offered traffic and the mean packet delay per node over the delivered traffic are plotted in Figures 10 and 11, respectively.

The variance of the throughput per node over the offered traffic continuously increases for the two CLDs as well as 802.11. However, the variance of the mean packet delay per node over the delivered traffic rapidly increases for values near to the saturated traffic of the 802.11 and the PBOA protocols. We define the saturated traffic as the aggregate throughput value that the protocols achieve if the inter arrival time approaches zero. Near to these traffic both schemes, the 802.11 as well as the power control-based PBOA CLD achieve high delivered traffic only by sacrificing fairness. The MUD-based CLD shows only a moderate increase of the variance of the mean packet delay per node, realizing the improved overall spectral efficiency in a fair manner.

The tendency that 802.11 and the power control-based CLD become unfair for high traffic load can also be observed by the Jain's fairness index described in Sect. VII. It is plotted for two offered traffic loads in Figure 12. While for an offered traffic of $3 \mathrm{Mbit} / \mathrm{s}$ all schemes achieve similar good fairness values, for high traffic 


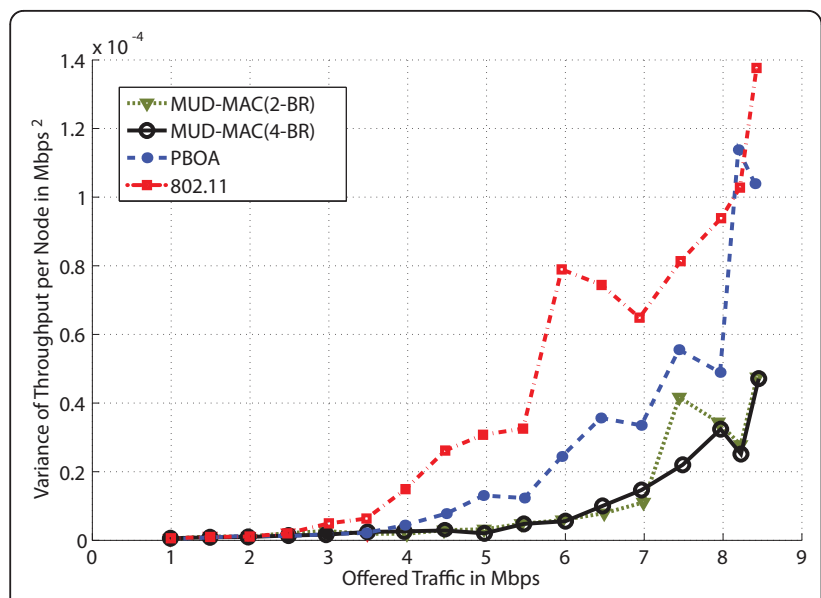

Figure 10 Variance of throughput per node over offered traffic for the 802.11, the MUD-MAC and the PBOA protocol with 50 nodes in a $500 \mathrm{~m} \times 500 \mathrm{~m}$ random network.

density (10 Mbit/s), the 802.11 protocol cannot even treat $60 \%$ of the users fair. PBOA is considerably fairer and handles $77 \%$ of the users equally. However, MUDMAC with both, two and four branches, shows the best fairness trends and can achieve a fair behavior for more than $89 \%$ (two branches) and $94 \%$ (four branches) of the users.

\section{Conclusions}

The goal of this work was a numerical comparison between two classes of CLDs that are both applied in the specific environment of ad hoc networks and aim at an increased spatial reuse compared to 802.11. The first class inherits all kinds of CLDs that use power control as a physical layer strategy to suppress MAI on the

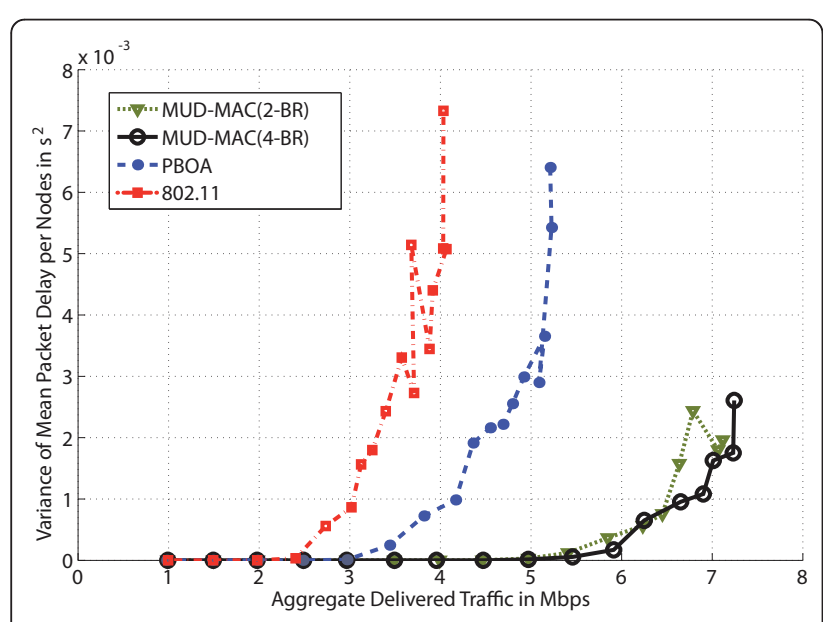

Figure 11 Variance of mean packet delay per node over delivered traffic for the 802.11, the MUD-MAC and the PBOA protocol with 50 nodes in a $500 \mathrm{~m} \times 500 \mathrm{~m}$ random network.

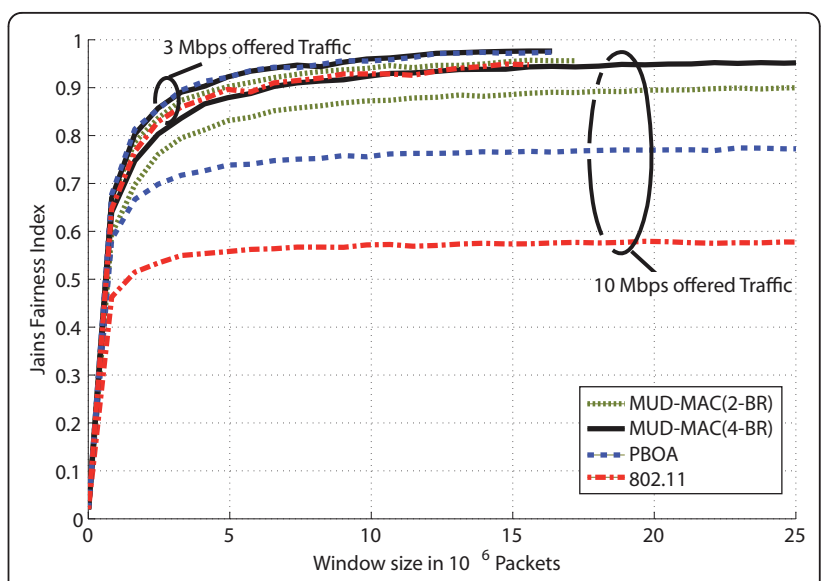

Figure 12 Jain's Fairness Index for offered traffic of $3 \mathrm{Mbit} / \mathrm{s}$ and $10 \mathrm{Mbit} / \mathrm{s}$ for the 802.11, the MUD-MAC and the PBOA protocol with 50 nodes in a $500 \mathrm{~m} \times 500 \mathrm{~m}$ random network.

transmitter side. In this work we gave a detailed summary of these methods and showed the shortcomings and advantages of the algorithms proposed in the literature.

The second class of cross-layer schemes assumes MUD on the physical layer. All algorithms within this class increase the spatial reuse by canceling interfering streams at the receiver side. We summarized methods proposed in the literature and pointed out their benefits and drawbacks.

For the simulative comparison we decided on the reference schemes by choosing in each case the most promising candidate out of the two classes. In order to investigate the QoS offered by the proposed CLDs, we evaluated the performance in terms of aggregate system throughput, delay, and fairness.

We showed by means of simulations that the MUDbased CLD not only is beneficial with respect to the aggregate system throughput compared to the power control-based CLD. Moreover, it can handle very dense interference situations that lead to a poor performance with the power control-based CLD.

We further found that the gains in throughput achieved by the MUD-based CLD do not originate from an unfair behavior that supports only favorable nodes at the cost of others. This is ensured by an increased fairness as well as better delay performance compared to the power control-based CLD. All above-mentioned results held also for low complexity multiuser detectors with only two branches.

\section{Abbreviations}

ACK: acknowledgment; Ann: announcement; CLD: cross-layer design; DATA: data transmission; DPC/ALP: distributed power-control algorithm with active link protection; FIFO: first in first out; MAC: medium access control; MAl: multiple access interference; MUD: multiuser detection; MIMO: multiple input 
multiple output; OBJ: objection; PER: packet error rate; PBOA: Progressive Backoff Algorithm; PHY: physical layer; QoS: quality of service; SINR: signal-tointerference-and-noise-ratio.

\section{Author details}

'Institute of Communication Networks, Technische Universität München, Arcisstr. 21, 80290 Munich, Germany ${ }^{2}$ DOCOMO Euro-Labs, Landsbergerstr. 312, 86687 Munich, Germany ${ }^{3}$ Institute IMDEA Networks, Avenida del Mar Mediterraneo, 2228918 Leganes (Madrid) Spain

\section{Competing interests}

The authors declare that they have no competing interests.

Received: 15 November 2010 Accepted: 8 June 2011

Published: 8 June 2011

\section{References}

1. A Muqattash, MM Krunz, "POWMAC: A Single-Channel Power-Control Protocol for Throughput Enhancement in Wireless Ad Hoc Networks". IEEE Journal on Selected Areas in Communications. 23(5):1067-1084 (2005)

2. IWH Ho, SC Liew, "Impact of Power Control on Performance of IEEE 802.11 Wireless Networks". IEEE Trans on Mobile Computing. 6(11):1245-1258 (2007)

3. S Toumpis, AJ Goldsmith, "New Media Access Protocols for Wireless Ad Hoc Networks Based on Cross-Layer Principles". IEEE Trans on Wireless Communications. 5(8):2228-2241 (2006)

4. S Verdu, Multiuser Detection. (New York, NY: Cambridge University Press, 1998)

5. S Verdu, Computational Complexity of Optimum Multiuser Detection". Algorithmica. 4(3):303-312 (1989)

6. V Kühn, R Böhnke, K Kammeyer, "Multi-user detection in multicarrier-CDMA systems". e \& i Elektrotechnik und Information-stechnik. 119, 395-402 (2002)

7. Y Eisenberg, K Conner, M Sherman, J Niedzwiecki, R Brothers, "MUD Enabled Media Access Control for High Capacity, Low-Latency Spread Spectrum Communications". Proc IEEE Military Communications Conference (MILCOM '07), Orlando, FL. (2007)

8. P Casari, M Levorato, M Zorzi, "DSMA: an Access Method for MIMO Ad Hoc Networks Based on Distributed Scheduling". Proc ACM/IEEE International Wireless Communications and Mobile Computing Conference (IWCMC '06), Vancouver, Canada. (2006)

9. K Kusume, R Vilzmann, A Müller, C Hartmann, G Bauch, "Medium Access in Spread Spectrum Ad Hoc Networks with Multiuser Detection". EURASIP Journal on Advances in Signal Processing - Special Issue on Cross-Layer Design for the Physical, MAC, and Link Layer in Wireless Systems. 1-14 (2009)

10. U Korger, K Kusume, C Hartmann, J Widmer, "Power Control versus Multiuser Detection based Cross-Layer Design in Ad Hoc Net-works". Proc IEEE International Symposium on Personal, Indoor and Mobile Radio Communications (PIMRC 2010). (2010)

11. U Korger, C Hartmann, K Kusume, J Widmer, "Quality of Service Oriented Analysis of Cross-Layer Design in Wireless Ad Hoc Networks". Proc 3rd International Workshop on Multiple Access Communications (MACOM 2010). (2010)

12. PC Ng, SC Liew, LB Jiang, "Achieving Scalable Performance in Large-Scale IEEE 802.11 Wireless Networks". Proc IEEE Wire-less Communications and Networking Conference (WCNC '05), New Orleans, LA. (2005)

13. V Kawadia, PR Kumar, "Principles and Protocols for Power Control in Wireless Ad Hoc Networks". IEEE Journal on Selected Areas in Communications. 23(1):76-88 (2005)

14. X.-H Lin, Y-K Kwok, VKN Lau, "A New Power Control Approach for IEEE 802.11 Ad Hoc Networks". Proc IEEE Int Symposium on Personal, Indoor and Mobile Radio Communications (PIMRC '03), Beijing, China. 2, 1761-1765 (2003)

15. A Muqattash, MM Krunz, "A Distributed Transmission Power Control Protocol for Mobile Ad Hoc Networks". IEE Transactions On Mobile Computing. 3, 113-128 (2004). doi:10.1109/TMC.2004.1

16. N Bambos, SC Chen, GJ Pottie, "Channel Access Algorithms with Active Link Protection for Wireless Communication Networks with Power Control". IEEE/ ACM Trans on Networking. 8(5):583-597 (2000). doi:10.1109/90.879345
17. JP Monks, V Bharghavan, WW Hwu, "A power controlled multiple access protocol for wireless packet networks". Proc of INFOCOM, Anchorage, AK, USA. (2001)

18. A Muqattash, MM Krunz, "CDMA-based MAC Protocol for Wireless Ad Hoc Networks". Proc ACM Int Symposium on Mobile Ad Hoc Networking and Computing (MobiHoc '03), Annapolis, MD. 153-164 (2003)

19. SHR Naqvi, LM Patnaik, "A Distributed Channel Access Protocol for Ad Hoc Networks with Feedback Power Control". IEEE Transactions on Mobile Computing. 5, 1448-1459 (2006)

20. $P$ Li, $X$ Geng, Y Fang, "An Adaptive Power Controlled MAC Protocol for Wireless Ad Hoc Networks". IEEE Transactions On Wireless Communications. 8, 226-233 (2009)

21. P Casari, M Levorato, M Zorzi, "MAC/PHY CrossLayer Design of MIMO Ad Hoc Networks with Layered Multiuser Detection". IEEE Trans on Wireless Communications. 7(11):4596-4607 (2008)

22. M Levorato, S Tomasin, M Zorzi, "Cooperative Spatial Multiplexing for Ad Hoc Networks with Hybrid ARQ: System Design and Performance Analysis". IEEE Trans Communications. 56(9):1545-1555 (2008)

23. J Zhang, Z Dziong, F Gagnon, M Kadoch, "Multiuser Detection Based MAC Design for Ad Hoc Networks". IEEE Transactions On Wireless Communications. 8, 1836-1846 (2009)

24. S Chu, X Wang, "Opportunistic and Cooperative Spatial Multiplexing in MIMO Ad Hoc Networks". IEEE Transactions On Networking. 18(5):1610-1623 (2010)

25. T Janevski, Traffic Analysis and Design of Wireless IP Networks. (Artech House Inc, 2003)

26. R Jain, D Chiu, W Hawe, "A quantitative measure of fairness and discrimination for resource allocation in shared computer systems". Digital Equipment Corporation, Tech Rep TR-301. (1984)

27. JG Proakis, Digital Communications. (McGraw-Hill Book Company, 1989)

\section{doi:10.1186/1687-1499-2011-9}

Cite this article as: Korger et al:: Quality of service implications of power control and multiuser detection-based cross-layer design. EURASIP Journal on Wireless Communications and Networking 2011 2011:9.

\section{Submit your manuscript to a SpringerOpen ${ }^{\mathcal{O}}$ journal and benefit from:}

- Convenient online submission

- Rigorous peer review

- Immediate publication on acceptance

- Open access: articles freely available online

- High visibility within the field

- Retaining the copyright to your article

Submit your next manuscript at $\gg$ springeropen.com 